\title{
Fast, High-Order Solution of Surface Scattering Problems
}

Oscar P. Brumo' and Leonid A. Kunwansky

Applied Mathematics. Caltech. Pasadena. CA 0125

Introduction. We present a new algorithm for the mumerical solution of problems of acoustic scattering by surfaces in three-dimensional space. This algorithm evaluates scattered ficlds through fast, high-order solution of the corresponding boundary integral equation. The high-order accuracy of onr solver is achieved through use of partitions of unity together with analytical resolution of kernel singularities. The acceleration, in turn, results from use of a novel approach which, based on high-order "two-face" equivalent source approximations, reduces the evaluation of far interactions to evaluation of 3-D FFT's. This ipproach is faster, substantially nore accurate, and it runs on dramatically lower memories than other FFT and $k$-space methods. The present algorithm computes one matrix-vector multiply in $\mathcal{O}\left(N^{0 / 5} \log N\right)$ tio $\mathcal{O}\left(N^{1 / 3} \log N\right)$ operations. where $N$ is the number of surface cliscretization points. The latter estinnate it]plies to smooth surfaces, for which our high order algorithm provides accurate solutions with small values of $N$ : the former: more favorable count is valicl for highly complex surfaces requiring significant anounts of subwavelength sampling. Further, our approach exhibits super-algebraic convergence, it can be applied to smooth and non-smooth scatterers, and, wnlike other accelerated schentes it docs not suffer from accuracy breakdowns of any kind (conpare $[6,10]$ and $[8, \mathrm{p} .576]$ ) In what follows we introduce the main algorithmic components in our approach and we demonstrate its performance with a variety of numerical results. In yarticular, we show that the present algorithm can evaluate accurately in a persontal computer scattering from bodies of acoustical sizes of several hundreds.

Mathematical Formulation. We consider a problem of acoustic scatticring by a sound-soft obstacle. This problem, governed by the Hehnholtz eguntion, cun be treated using the acoustic single- and double- layer potentials (sce e.g. [7])

$$
(S \varphi)\left(\mathbf{r}^{\prime}\right)=\int_{\partial D} \Phi\left(\mathbf{r}^{\prime}, \mathbf{r}\right) \varphi(\mathbf{r}) d s(\mathbf{r}) \text { and }\left(K_{\varphi}\right)\left(\mathrm{r}^{\prime}\right)=\int_{\partial D} \frac{\partial \Phi\left(\mathbf{r}^{\prime}: \mathbf{r}\right)}{\partial \nu(\mathbf{r})} \varphi(\mathbf{r}) d s(\mathbf{r})
$$

Here $\Phi\left(\mathbf{r}^{\prime}, \mathbf{r}\right)=e^{i k\left|\mathbf{r}^{\prime}-\mathbf{r}\right|} / 4 \pi\left|\mathbf{r}^{\prime}-\mathbf{r}\right|$ is the Cireen function for the Helmloltiz cxplurtion, and $\nu(\mathbf{r})$ is the external normal to the surface $\partial D$ at point $r$. Explicitly; given the values of the incoming wave $\psi^{i}(\mathbf{r})$ on $\partial D$, the scattered ficld can be: obtained easily once the integral equation for the unknown density $\varphi(r)$

$$
\frac{1}{2} \varphi(\mathbf{r})+(K \varphi)(\mathbf{r})-i n(S \varphi)(\mathbf{r})=\psi^{i}(\mathbf{r}), \quad \mathbf{r} \in \partial D
$$

has been solved. Naturally, the pussibility of producing fast and accurate solutions for our problems linges on our ability to evaluate the integrals (1) accurately

${ }^{1}$ Presenter and corresponding anthor. 
and efficiently. In atternpting to develop such accurate and efficient integrators one faces two main problems, namely, accurate evaluation of the singular adjacent interactions - without undue compromise of speed - and fast evaluation of the voluminous number of nonadjacent interactions - without compromise in accuracy. In what follows we present a solution to these problems.

Partitions of unity. In order to deal with topological characteristics of closed surfaces which are given in terns of local parametrizations we utilize partitions of unity. In detail, we use a covering of the surface $\partial D$ by a number $K$ of overlapping two-dimensional patches $\mathcal{P}^{j}, j=1, \cdots, K$, (called local charts in differential geometry). The patches $\mathcal{P}^{j}$ are then smoothly mapped to coordinate sets $\mathcal{H}^{j}$ in two-dimensional space, where actual integrations are performed. Further, we utilize a partition of unity subordinated to this covering of $\partial D$, i.e. we introduce a set of non-negative smooth functions $\left\{w^{j}, j=1, \ldots, K\right\}$, such that (i) $w^{j}$ is defined, smooth and non-negative in $\partial D$, and it vanishes outside $\mathcal{P}^{j}$, and (ii) $\sum_{j=1}^{K} w^{j}=1$ throughout $\partial D$. This allows us to reduce the problem of integration of the density $\varphi(r)$ over the surface to a calculation of integrals of smooth functions $\varphi^{j}$ compactly supported in the planar sets $\mathcal{H}^{j}$.

Adjacent integration. Substantial difficulties in the high-order evaluation of adjacent interactions are caused by the singular nature of the integral kernels. While, certainly, the well-known strategy of "singularity subtraction" gives rise to bounded integrands, integration of such bounded functions by means of classical high-order methods does not exhibit high-order accuracy - since the subsequent derivatives of the integrand are themselves unbounded. The new basic highorder integrator we present is based on analytical resolution of singularities. The resolution is achieved by integration in polar coordinates centered around each singular point. The Jacobian of the corresponding change of variables has the effect of cancelling the singularity, so that high order integration in the both radial and angular directions can be performed using the trapezoidal rule. Since the corresponding radial quadrature points do not lie on the Cartesian grid, a high-order, fast interpolation technique has been developed for evaluation of the necessary function values at the radial integrations points. Efficiency is of utmost importance here, since we use one such polar coordinate transformation at each target point. Our high order integrator exhibits super-algebraic convergence for smooth and non-smooth scattering surfaces [1-3].

Non-adjacent integration and acceleration. Our accelerator is closely related to two of the most advanced FFT methods developed recently $[4,9]$. An important common element between these two methods and our technique is a concept of equivalent (or auxiliary) sources, located on a subset of a 3-D Cartesian grid. In all three cases, the intensities of these sources are chosen to approximate the field radiated by the scatterer, which allows for fast computation of the "nonadjacent interactions" through the use of 3-D FFTs. Surface problems like the ones we consider are treated in $[4,9]$ by means of equivalent sources located in a volumetric grid - in such a way that equivalent sources with non-zero intensities occupy all Cartesian nodes adjacent to the scatterer. Since the spacing of this Cartesian grid cannot be coarsened beyond some threshold for surface 
problems such a scheme requires a $\mathcal{O}\left(N^{3 / 2}\right)$ FFT. Therefore, previous FFT surface scattering solvers require $\mathcal{O}\left(N^{3 / 2}\right)$ units of RAM and they run in $\mathcal{O}\left(N^{3 / 2} \log N\right)$ operations. Our algorithm, in contrast. subdivides the volume occupied by the scatterer into a number of (relatively large) cubic cells, and it places equivalent sources on the faces of those cells. As we have shown, such a design reduce significantly the sizes of the required FFTs - to as little as $\mathcal{O}\left(N^{6 / 5}\right)$ to $\mathcal{O}\left(N^{4 / 3}\right)$ points - with proportional improvement in storage requirenents and operation count. Further, it results in super-algebraic convergence of the equivalent source approximations as the size of the scatterer is increased.

Resolution of singularities To obtain resolution of the singular integrands around the ogive's conical singularities, for example: a combination of two changes of variables were used: a polar change of variables similar to that described in the section "Adjacent integration" above, followed by i polynomial change of variables which regularizes the Hölder-type singularity of the turlerlying density see [3] for details.

Numerical Results. We present results for two well known and widely used test geometries: large ellipsojds and ogives. Solutions of the linear systens arising from discretization of equations (2) were obtained in all cases by mean of a version of the iterative solver GMRES. In all cases we used the value $\gamma=$ $\max \{3, A / \lambda\}$ for the coupling constant in equation (2) - where $A$ is the diameter of the scatterer. We have found that this value of $\gamma$ leads to a substantially reduced number of GMRES iterations. All computations were performed on a $400 \mathrm{MHz}$ Pentium II processor and with $1 \mathrm{~Gb}$ of RAM. In the following tables we used boundary conditions as given by a unit source located inside the ogive for which the exact solution is known: it equals the field radiated by the source Convergence studies for plane-wave boundary conditions showed errors contistent with those displayed in these tables. The symbols $\varepsilon_{2}$ and $\varepsilon_{\infty}$ denote the far field errors in the relative mean-square norm and the absolute maximum norm, respectively

\begin{tabular}{|c|c|c|c|c|c|c|}
\hline Size & $\# \mathbf{I t}$ & T/it & RAM & Unknowns & $\varepsilon_{\infty}$ & $\varepsilon_{2}$ \\
\hline \hline $80 \lambda \times 20 \lambda \times 20 \lambda$ & 15 & $5 \mathrm{~h} 22 \mathrm{~m}$ & $600 \mathrm{M}$ & 691206 & $1.4 \cdot 10^{-4}$ & $2.9 \cdot 10^{-5}$ \\
\hline $100 \lambda \times 25 \lambda \times 25 \lambda$ & 15 & $5 \mathrm{~h} 29 \mathrm{~m}$ & $600 \mathrm{M}$ & 691206 & $1.1 \cdot 10^{-3}$ & $2.4 \cdot 10^{-4}$ \\
\hline
\end{tabular}

Table 1: Scattering from large ellipsoids; point source inside the body.

Table 1 presents results for problems of scattering by very large ellipsoids; note the excellent accuracies provided by the algorithm in competitive running times. Table 2 displays a set of results obtained for scattering from a singular surface: the ogive depicted in Figure 1. for acoustical sizes (distances between tips) equal to $1 \lambda, 10 \lambda$ and $20 \lambda$. For the larger sizes we used the accelerator deșcribed above; not the substantial improvements in computing times resulting from the acceleration algorithm.

Acknowledgment and Disclaimer: Effort sponsored by the AFOSR under grant numbers F49620-96-1-0008 and F 49620-99-1-0010. 


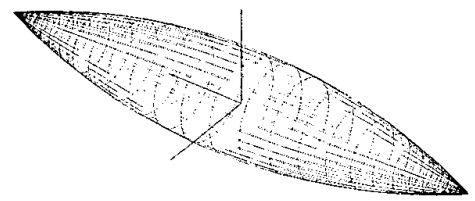

Figure 1: Ogine geometry puesented in werenes [11].

\begin{tabular}{|c|c|c|c|c|c|c|}
\hline Type & Size & Uliknow1:s & Tterations & Thime/It. & $\varepsilon_{\infty}$ & $\varepsilon_{2}$ \\
\hline Non $A$ cocelerated & $1 \lambda$ & 1568 & 20 & $69 \mathrm{~s}$ & $2.5 \times 10^{-3}$ & $1.4 \cdot 10^{-3}$ \\
\hline Non $A$ crelomed & $1 \lambda$ & 6336 & 17 & $12 \mathrm{~m} 45 \mathrm{~s}$ & $3.8 \times 10^{-5}$ & $2.2 \cdot 10^{-5}$ \\
\hline Non Accelcrated & $1 \lambda$ & 25472 & 17 & $3 \mathrm{~h} 27 \mathrm{~m}$ & $9.8 \times 10^{-7}$ & $4.8 \cdot 10^{-7}$ \\
\hline Accolerated & $10 \lambda$ & 34112 & 13 & $26 \mathrm{~mm}$ & $3.8 \times 10^{-4}$ & $2.1 \cdot 10^{-4}$ \\
\hline Accelerated & $20 \lambda$ & 34112 & 1.4 & $14 \mathrm{~m}$ & $6.0 \times 10^{-3}$ & $2.4 \cdot 10^{-3}$ \\
\hline Actolemates & $20 \lambda$ & 72320 & 19 & $67 \mathrm{~m}$ & $5.4 \times 10^{-5}$ & $2.1 \cdot 10^{-5}$ \\
\hline
\end{tabular}

Tible: 2: Scattering by an ogive.

\section{References}

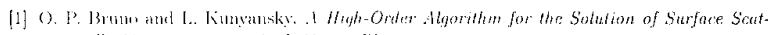

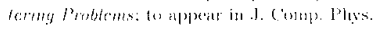

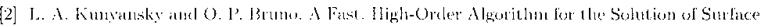

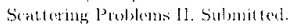

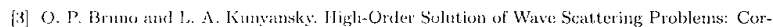

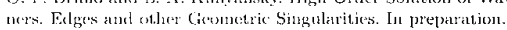

[1] F. B3l

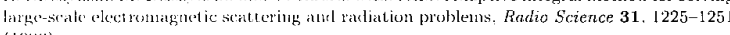
$(19 !)(i)$.

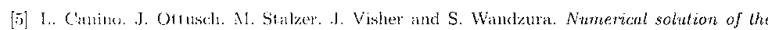

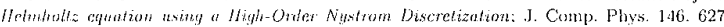
$6(6 ; 3)(19 \%)$

[6] B. Dembart and E. Yip, The accrracy of Fust Multipole Methods for Moxwell's Equations 1FI:E Compunational Science and Engineering: 18-56. July-September 1998

[7] D. Cotiton wnd R. Kress. Inwerse acoutstic and electromagnetic scattering theory. SpringerVerlag. 1992 .

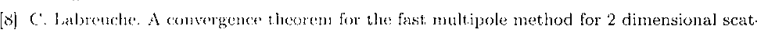

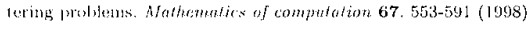

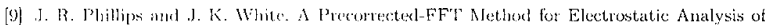

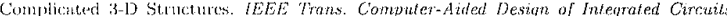

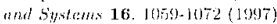

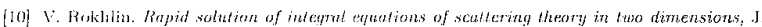
Comp. 1) 19: $86, .114-130.1990$.

[11] A. C. Woo. H. T. (. Wamb. M. I. Scluhh and M. L. Saunders, Benclunark Radar Tar-

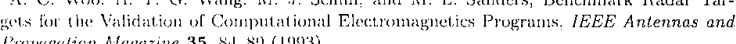

